\title{
Deracemization through Concurrent Oxidation and Reduction
}

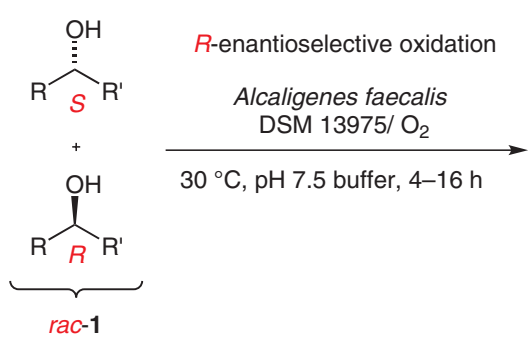

Selected examples:
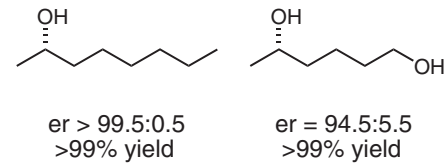

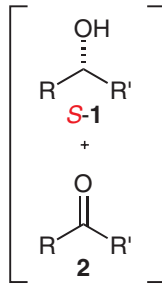
$>99 \%$ yield
S-stereoselective reduction
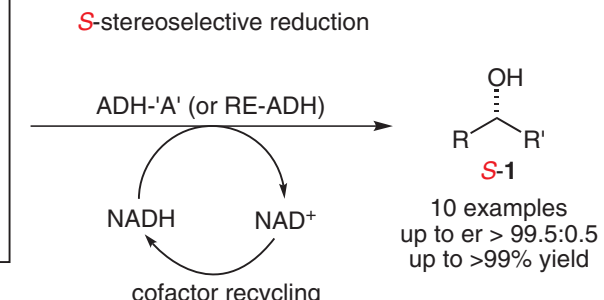

(glucose dehydrogenase (GDH)/ glucose)

ADH-'A': alcohol dehydrogenase from Rhodococcus ruber DSM 44541 RE-ADH: alcohol dehydrogenase from Rhodococcus erythropolis

Significance: Biocatalytic deracemization of secalcohols rac-1 has been achieved through a concurrent stereoselective oxidation-reduction sequence affording the S-enantiomer $S$-1 in highly enantioenriched form (up to er > 99.5:0.5). The authors have identified resting cells of Alcaligenes faecalis DSM 13975 as a highly active and $R$ enantioselective catalyst for the oxidation of secalcohols. Molecular oxygen serves as oxidant. In the reduction step, an $S$-selective alcohol dehydrogenase, either ADH- 'A' or RE-ADH, in combination with a cofactor recycling system (glucose/ $\mathrm{GDH}$ ) was effective. The method has also been applied to the stereoinversion of an enantiopure $\mathrm{sec}$-alcohol. A related system gives access to the $R$-enantiomer through $S$-enantioselective oxidation and $R$-stereoselective reduction.
Comment: Dynamic kinetic resolution of sec-alcohols through the racemization of the alcohol moiety in combination with an (enzymatic) kinetic resolution is a well-established strategy. For the deracemization of alcohols through enantioselective oxidation and simultaneous stereoselective reduction of the corresponding ketones no general protocol has been described due to the lack of an applicable sec-alcohol oxidase. In the present work, two examples highlight the use of 2-propanol as an alternative cofactor recycling system resulting in only slightly reduced enantioselectivities. Both enantiomeric forms of the secalcohol are accessible. The $R$-enantiomer, howev$\mathrm{er}$, is obtained with generally lower enantioselectivities. 